\title{
« Dans le principe, les idées vraies servent toujours le peuple ». Science et émancipation chez Althusser
}

Jean Matthys

\section{OpenEdition}

\section{Journals}

Édition électronique

URL : http://journals.openedition.org/grm/585

DOI : $10.4000 / \mathrm{grm} .585$

ISSN : 1775-3902

\section{Éditeur}

Groupe de Recherches Matérialistes

\section{Référence électronique}

Jean Matthys, « «Dans le principe, les idées vraies servent toujours le peuple». Science et émancipation chez Althusser », Cahiers du GRM [En ligne], 7 | 2015, mis en ligne le 05 juin 2015, consulté le 30 avril 2019. URL : http://journals.openedition.org/grm/585 ; DOI : 10.4000/grm.585

Ce document a été généré automatiquement le 30 avril 2019

(C) GRM - Association 


\title{
"Dans le principe, les idées vraies servent toujours le peuple ». Science et émancipation chez Althusser
}

\author{
Jean Matthys
}

\section{Pour une réévaluation du théoricisme comme stratégie politique}

Il semble convenu, lorsqu'il s'agit de lire et "sauver Althusser ", de creuser toujours davantage le fossé de l'autocritique. Une première manière de ce travail de fossoyeur, qui peut s'autoriser de l'auctoritas d'Althusser lui-même, consiste à reléguer la période dite "théoriciste» dans un passé inavouable, à jamais marqué du sceau d'une conjoncture dépassée. Le «vrai » Althusser - comprenez : ce qu'il y a d'appropriable dans sa pensée ne commencerait qu'après l'autocritique - processus entamé, rappelons-le, dès 1967, et culminant dans les Éléments d'autocritique de 1974. La version la plus radicale de cette lecture a abouti le plus souvent à une surévaluation des écrits du «matérialisme aléatoire » du dernier Althusser dont G. M. Goshgarian rappelait récemment, et à juste titre, combien sa production « reste une goutte dans la mer $»^{1}$. La seconde approche, apparemment opposée mais, en cela, solidaire de la première, consiste à chercher systématiquement à montrer à quel point les thèmes post-autocritiques se trouvaient en réalité déjà bien présents, ne fût-ce que sous forme de tendance, dans les premiers écrits. Si cette seconde approche a le mérite de vouloir faire droit au premier Althusser, elle semble n'y arriver qu'au prix d'un double jeu de reconnaissance/méconnaissance à l'égard de l'autocritique, puisqu'il s'agit d'en atténuer le caractère de " coupure », tout en important systématiquement les concepts ultérieurs à l'aune desquels les premiers écrits doivent être lus et jugés. C'est dire combien ces deux approches sont, à leur manière, profondément hégéliennes: dans les deux cas, la vérité de l'althussérisme se trouve au terme de son devenir, offrant l'image d'un Telos vers lequel tout tendait toujours-déjà dès 
l'Origine. Et si ces deux approches complices se fourvoyaient, du fait même qu'elles soutiennent toutes deux, quoiqu'à des degrés divers, qu'il faut réussir à penser un «Althusser sans le théoricisme »? Et si la contre-courbure qu'elles ont imposée devait aujourd'hui être rectifiée, afin de réactiver le sens politique de la « déviation théoriciste » ${ }^{2}$ originaire $^{3}$ ?

2 De fait, ces deux lectures rétrospectives, aboutissant souvent à une forme de spontanéisme de la multitude s'autorisant d'une ontologie (sinon d'une mystique) de l'aléatoire, ont pu avoir tendance à occulter le cœur du projet althussérien en tant qu'il visait dans son intégralité tout à la fois à problématiser et à resserrer le lien entre théorie et pratique d'émancipation. Le principe de l'autocritique consiste à dénoncer le théoricisme antérieur compris comme "primat de la théorie sur la pratique ; insistance unilatérale sur la théorie $»^{4}$. Dénonçant le phantasme rationaliste et idéaliste de la toutepuissance des idées, l'autocritique ne remet pas pour autant en question comme telle l'idée d'une efficace et d'une autonomie, relatives mais bien réelles, de la théorie. En dernière instance, l'autocritique semble en réalité se limiter à l'introduction d'une clause exigeant une réévaluation proprement matérialiste de la coupure marquant la naissance inédite et sans fin d'une "science révolutionnaire ", afin d'" expliquer les conditions, la raison et le sens de ce couple inouï, qui fait "bouger" quelque chose de décisif dans notre idée de la science $»^{5}$.

On n'a pas assez remarqué que la question des conditions concrètes d'émergence de la science marxiste est en réalité déjà effleurée dans ce bref passage de Lire Le Capital, quoique laissée en friche :

Contentons-nous de retenir qu'il faut que le sujet ait occupé dans le nouveau terrain sa nouvelle place, autrement dit que le sujet ait déjà été, voire partiellement à son insu, installé sur ce nouveau terrain, pour pouvoir porter sur l'ancien invisible le regard instruit qui lui rendra visible cet invisible ${ }^{6}$.

Cette thèse reste sans suite dans le texte de 1965, d'autant que son élucidation matérialiste entre en conflit avec le refus althussérien de récuser "sans égard » la thèse kaustkyste-léniniste de l'importation de la théorie marxiste depuis une pratique théorique extérieure au prolétariat - récusation qu'il range alors aux côtés des thèses spontanéistes, gauchistes et humanistes ${ }^{7}$. Cet « insu » pratique et politique déterminant la possibilité pour Marx de rompre théoriquement avec l'idéologie bourgeoise ne sera analysé par Althusser que dans les années 1970, dans des textes prenant dès lors clairement position contre la théorie kautskyste ${ }^{8}$.

Quoi qu'il en soit, loin de voir dans l'introduction de la clause autocritique un changement radical de perspective, il faut n'y voir à la limite qu'un simple déplacement de focale, qui peut s'autoriser de ce "contentons-nous" prudent autant que programmatique. La présence en creux de ce thème dès 1965 nous révèle qu'il est possible de maintenir une forme de «théoricisme » tout en y adjoignant la clause matérialiste. Celle-ci renvoie à l'étude des conditions de possibilité de l'émergence (surdéterminée) du voir scientifique, mais ne rend pas obsolète le théoricisme entendu comme thèse politique soutenant que la science produit, en principe, des effets émancipateurs au sein de la lutte des classes. Les deux thèses: "sans théorie révolutionnaire, pas de mouvement révolutionnaire » et "sans mouvement révolutionnaire, pas de théorie révolutionnaire " sont bien "unies comme les dents et les lèvres " ${ }^{9}$. Jamais, en effet, Althusser n'abandonnera l'idée d'une lutte de classe dans la théorie, et de la théorie comme enjeu et 
forme d'existence de la lutte des classes - comme le révèle cet extrait justement tiré des Éléments d'autocritique :

Reprendre et défendre, dans ce contexte et ce programme, le mot «science » : nous le devons contre tous les idéalistes subjectifs bourgeois, et contre les marxistes petits-bourgeois, qui, les uns et les autres, crient à son simple énoncé au "positivisme", sans doute parce que la seule représentation qu'ils peuvent ou veulent se faire de la pratique et de l'histoire d'une science, et $a$ fortiori de la science marxiste, est la représentation positiviste classique ou vulgaire, bourgeoise ${ }^{10}$.

Dès Pour Marx et Lire "Le Capital », le programme "théoriciste » n’a jamais consisté à appliquer ou plaquer une conception a priori de la science sur la pratique révolutionnaire, mais à repenser en profondeur, et à la hauteur de ce qu'exigeait la fusion marxiste de la théorie et de la pratique - cet événement historique sans précédent -, le concept même de scientifique. Nombre de faux procès qui ont été faits à l'althussérisme n'ont pas tiré cette leçon essentielle qui constitue pourtant le cœur de sa stratégie philosophique. Si, comme le rappelait récemment É. Balibar, l'entreprise d'Althusser, inséparablement théorique et politique, consistait à "transformer le concept de science " ${ }^{11}$, il nous semble utile de repenser une forme positive, quoique problématique, de « théoricisme».

\section{Science et émancipation : première tentative d'articulation}

6 Dans un petit texte de 1968 à vocation militante et intitulé « La philosophie comme arme de la révolution", Althusser soutient que "Dans le principe, les idées vraies servent toujours le peuple ; les idées fausses servent toujours les ennemis du peuple $»^{12}$. Rarement Althusser aura formulé aussi explicitement " la thèse selon laquelle le savoir a, en luimême, une valeur subversive à l'égard de la domination bourgeoise ${ }^{13}$. Cependant, contrairement à son disciple dissident Jacques Rancière, je voudrais y voir l'indice d'un problème à poser et à penser, plutôt qu'une posture platonicienne technocratique à discréditer d'emblée. Derrière la forte teneur idéologique et polémique de cette thèse qui pourrait être tout droit tirée du Petit livre rouge, à quoi son auteur nous invite-t-il à penser?

7 Une remarque préliminaire. Dogmatique, cette thèse l'est sans l'ombre d'un doute, mais dans un sens tout à fait assumé par Althusser : celui de la prise de position par position de thèse. Dans la soutenance d'Amiens, Althusser affirme qu'une thèse philosophique est forcément extrême, flirtant toujours avec une forme d'« excès ». Poser des "thèseslimites ", à la limite du pensable ou du tenable, "penser aux extrêmes » et "occupe[r] la place de l'impossible» "pour rendre la pensée possible $»^{14}$ : c'est ainsi qu'Althusser caractérise le geste philosophique matérialiste. Illustrant cette idée par la référence à Machiavel et son Prince absent pour un État inexistant - et nous pourrions ajouter le Dieu-vide de Spinoza ${ }^{15}$-, Althusser nous fournit une première indication précieuse pour essayer de comprendre à la fois son propre geste lorsqu'il affirme cette thèse, et le contenu de celle-ci, en tant qu'elle fait signe, on le verra, vers une forme de " faire le vide ${ }^{16}$.

Pour Althusser, la coupure entre science et idéologie, vérité et erreur, recoupe la ligne de fracture tracée par la lutte des classes. "C'est le même mot. Ligne de démarcation théorique entre les idées vraies et les idées fausses. Ligne de démarcation politique entre le peuple (le prolétariat et ses alliés) et les ennemis du peuple $\aleph^{17}$. Althusser refuse ainsi le relativisme de classe qu'implique la théorie lyssenkiste des deux sciences. Il n'y a pas une 
science bourgeoise et une science prolétarienne ; la science est du côté du prolétariat et de son émancipation. Or, s'il refuse la distinction entre science bourgeoise et science prolétarienne, mais qu'il maintient néanmoins un lien serré entre la science et la politique d'émancipation, il faut alors qu'il y ait nécessairement quelque chose « dans » la science elle-même qui ait intrinsèquement à voir avec l'émancipation des sujets. Toute la question est de comprendre ce qu'est la science pour Althusser, et ce qu'il y a d'émancipateur « en » elle.

9 La piste à explorer me semble être la suivante : la science, telle que la pense Althusser, produit des effets de désubjectivation, par où s'ouvrent de nouvelles voies de subjectivation, en lien avec de nouvelles formes de vie et d'organisation. Ces effets tiennent à plusieurs caractéristiques essentielles de la science althussérienne, que l'on peut ramasser en une expression schématique sans doute trop simple, mais qui peut au moins servir de mot d'ordre provisoire : la science telle que la pense Althusser est une science de la complexité irréductible, à la fois intelligible (par la pratique théorique) et transformable (par la pratique politique). L'expression « science de la complexité » inclut en fait deux niveaux, en fonction du sens du génitif qui peut être (1) subjectif ou (2) objectif.

1. Du point de vue de sa forme ou de sa structure productive, la science est elle-même une pratique complexe qui opère par transformation conceptuelle d'un donné idéologique, sans garantie ou norme extrinsèque, et sans faire directement appel aux sujets comme tels $s^{18}-\mathrm{y}$ compris et surtout sous la forme d'un sujet supposé savoir. Inscrite dans ce tout réel complexe dont elle veut produire la connaissance, la science se présente comme un interminable travail de production d'idées vraies, sans Origine, ni Sujet, ni Fin. Néanmoins, en tant que pratique asubjective de transformation des représentations idéologiques qui en constituent la matière première, elle touche au cœur du «monde vécu » des sujets. On peut parler ici d'une voie épistémologique, ou de théoricisme comme thèse politique : la science est comme telle - de par sa nature, sa structure, sa forme, et en vertu de ce qu'elle « fait » aux sujets dans l'acte même de produire des idées vraies - émancipatrice.

2. Du point de vue de son objet, la science produit une connaissance du «tout complexe structuré à dominante » qu'elle vise à rendre intelligible sans en réduire la complexité. Elle est une science dont l'objet n'est autre que la complexité du tout social qui agit par surdétermination ou causalité structurale immanente, dont il faut étudier la structure et les instances «en conjoncture", sans jamais pouvoir les réduire à une essence simple et univoque, qu'elle soit spirituelle ou économique, originaire ou téléologique. On peut parler ici d'une voie ontologique soutenant que les idées vraies sont émancipatrices en vertu de leur objet, qui n'est autre que la complexité du tout social conflictuel qu'elles visent à rendre intelligible, et transformable à travers l'action politique réfléchie.

10 Même si l'ontologique finira par dominer les écrits tardifs du matérialisme aléatoire, ces deux voies coexistent du début à la fin de l'œuvre d'Althusser. C'est que, loin d'être séparées, elles sont intimement liées, au point de tracer une forme de cercle qui caractérise toute la théorie de la coupure épistémologique. D'un côté, c'est parce qu'elle fait droit à la complexité irréductible de la structure sociale, donc parce qu'elle vide la prétention de toute instance à se présenter comme le Centre unique ou l'essence expressive de cette structure, que la science déconstruit et transforme les représentations idéologiques des sujets qui tendent spontanément à se structurer autour d'un Centre, d'une Origine, d'un Telos, d'un grand Sujet en relation spéculaire avec ses petits sujets. Mais en même temps et à l'inverse, c'est parce qu'elle constitue une expérience asubjective, ou plutôt une pratique désubjectivante, qu'elle peut produire la connaissance de la structure sociale dans toute sa complexité - en ce compris les espaces, les 
temporalités et les tendances sous-déterminées, dominées, invisibilisées, où la science marxiste doit prendre position, politiquement et théoriquement, pour être en mesure de « voir ». À cet égard, s'il y a une forme de privilège épistémique accordé à la position théorico-politique de classe prolétarienne, ce n'est pas parce qu'elle fournirait un point de vue assurant un savoir total ou totalisant, mais au contraire parce que le prolétariat est ce qui, par son existence même, tout à la fois exige et permet la négation de tout projet théorique totalisant qui ne peut être par définition qu'idéologique, jamais scientifique.

11 Ainsi, ce qui fonderait la confiance althussérienne, ou plutôt sa pure et simple redéfinition de la science comme intrinsèquement émancipatrice, peut se comprendre à partir de cette double piste : du point de vue de sa structure, la science est un procès sans sujet, une activité désubjectivante ; et du point de vue de son objet, elle est une science de la topique sociale, dont les instances sont distinctes et en rapports complexes de dépendance relative, de manière à constituer un tout intelligible et transformable - la condition de possibilité de cette transformation politique résidant justement dans le caractère irréductiblement complexe du tout social composé d'instances réellement hétérogènes produisant, par voie de surdétermination, ce que nous pourrions appeler, à la suite d'Althusser, du «tout autre $»^{19}$. Dans l'idée de renouer avec le sens du théoricisme comme stratégie politique, la suite de la présente contribution sera consacrée à l'étude de la structure de la pratique théorique et de son rapport à l'émancipation.

\section{De la structure de la pratique théorique comme "procès sans sujet »}

12 Pour Althusser, « tout discours scientifique est par définition un discours sans sujet, il n'y a de "Sujet de la science" que dans une idéologie de la science $»^{20}$. Le procès de connaissance est, comme toute pratique, une production, c'est-à-dire la transformation d'une matière première donnée en un produit spécifique, à l'aide de moyens de production. Dans le cadre de la pratique théorique, les trois éléments constitutifs de sa structure sont tous des " généralités ", c'est-à-dire des " concepts généraux ", des "représentations " abstraites $^{21}$. Si ce procès, en tant qu' « appareil de pensée, fondé et articulé dans la réalité naturelle et sociale $»^{22}$ peut bien avoir des $"$ agents $»^{23}$, il est rigoureusement sans sujet. Plus exactement, si quelque chose comme du « sujet » est requis et produit par le procès, c'est en tant qu'effet et jamais comme origine, créateur ou garant d'un Telos: dans la pratique théorique, «c'est le système défini des conditions de la pratique théorique qui assigne à tel ou tel sujet (individu) pensant sa place et sa fonction dans la production de connaissances $»^{24}$. Selon É. Balibar, il en va chez Althusser de la pratique théorique comme de toute forme de pratique sociale-historique: son "pseudo-sujet est une structure, une complexité de conditions de possibilité qui "distribuent" et "disposent" les pratiques les unes par rapport aux autres $»^{25}$.

13 C'est dire immédiatement qu'Althusser, coupant court aux accusations de technocratisme que l'on a pu lui faire ${ }^{26}$, refuse d'accorder à un prétendu « Sujet de la science » quelque statut de pouvoir ou de savoir particulier. Non pas parce que la connaissance scientifique serait nulle, mais parce qu'elle est comme telle un processus sans sujet. Ce procès sans sujet, qui est une activité, destitue immédiatement le «scientifique » de sa position de «sujet supposé savoir». Mais en outre, au niveau de son résultat ou produit, elle 
transforme les représentations idéologiques constitutives du monde vécu des sujets : c'est en ce sens qu'il faut entendre qu'elle dotée d'une puissance désubjectivante. La science est donc sans Sujet, ni sujets-assujettis : ce sont les concepts articulés en une problématique théorique ${ }^{27}$ qui pensent. En ce sens, le « subjectif sans sujet ${ }^{28}$ en jeu dans la pratique scientifique, c'est précisément l'expérience de dessaisie de soi comme sujet de la part de ses agents. C'est la science qui pense, entendue comme logique du déploiement des concepts ou encore comme pratique scientifique de la démonstration, produisant des concepts neufs (Généralité III) en transformant d'anciennes notions idéologiques (Généralité I) par le «travail » de la Généralité II. Les idées vraies produites par le procès de connaissance constituent une "totalité-de-pensée », un « concret-de-pensée » défini comme "produit du penser, du concevoir (Produkt des Denkens, des Begreifens) $»^{29}$. Les sujets-agents n'en sont que le « lieu », les supports (Träger), jamais l'origine ou les garants.

Mais comment une science sans sujet, qui se constitue par la trop célèbre «coupure épistémologique ", peut-elle être en contact avec l'idéologie des sujets dont elle doit permettre une forme de désubjectivation? Pas plus que celui des autres, le discours de la science n'est jamais pur et ce, en vertu (1) de sa matière première, constituée de notions idéologiques antérieures; (2) de la forme de ses éléments constitués d'abstractions idéelles que partage l'idéologie qui fait feu de tout bois (idées, discours, pratiques matérielles, rituels, sentiments, impressions, objets, images, "directions », etc. $\left.{ }^{30}\right)$; (3) de ses agents-supports qui, s'ils ne sont pas mobilisés comme tels dans le procès de connaissance, n'en sont pas moins sujets idéologiques par ailleurs. Autrement dit, l'avantage avec l'idéologie, ce qui fait sa force et sa faiblesse, c'est qu'on la retrouve partout. Par conséquent, il n'y a aucune raison de craindre que l'idéologie soit coupée du monde (puisqu'elle constitue précisément le monde même des sujets), et donc qu'elle soit coupée des autres pratiques, y compris la pratique scientifique. Par conséquent et inversement, cette dernière ne peut être absolument coupée de l'idéologie, quoiqu'elle y tende par essence, par où est toujours maintenue ouverte la possibilité d'une efficace idéologique des idées de la science sur les sujets.

Notons que pour Althusser, soucieux de faire droit à la lutte de classe tout en sauvegardant l'autonomie relative de la science et en maintenant la thèse de la coupure ${ }^{31}$, c'est plus précisément à la philosophie qu'incombe la tâche d'articuler la théorie et la politique. C'est par la médiation de la philosophie que se produit la politisation de la théorie, son passage "de la forme-théorie à la "forme-idéologie" $»^{32}$, condition de son efficace pratique sur les sujets et l'histoire. La philosophie devra cependant constamment veiller à ce que ce devenir-idéologique ne vienne pas affecter la science comme telle, toujours menacée d'ossification dogmatique, d'hypostasiation et de respécularisation autour d'un Sujet de la science ou de l'Histoire. La philosophie remplit ainsi une fonction de "tierce instance $»^{33}$, permettant de penser l'efficace de la science dans et sur la politique ("pas de politique révolutionnaire sans un moment théorique») et de la politique sur la science (" pas de théorie révolutionnaire sans pratique politique »), tout en préservant l'autonomie relative de la pratique scientifique.

Voilà donc ce qui fonderait la confiance althussérienne dans les effets émancipateurs de toute science : parce que, par définition, «dans le principe », de par son mécanisme même, la science implique un effet de désubjectivation, de décentrement, de déspécularisation et, tendanciellement, de désassujettissement dans la structure d'interpellation idéologique ${ }^{34}$. Une difficulté persiste néanmoins: si l'on voit relativement bien en quoi cette thèse vaut dans le cas de la science marxiste de l'histoire et des formations sociales, 
il est moins évident de saisir en quoi toutes les sciences, toutes les « idées vraies servent toujours le peuple». Sans pouvoir suffisamment développer ce point ici, il me semble utile de « creuser » dans la direction suivante.

Les trois découvertes scientifiques majeures sont pour Althusser : les mathématiques au $\mathrm{V}$ ${ }^{e}$ siècle avec Thalès (suivie de la fondation de la philosophie avec Platon), la physique mathématique moderne au XVII ${ }^{e}$ siècle avec Galilée (produisant des répercutions philosophiques chez Descartes) et le "continent-Histoire" au XIX siècle avec MarX (suivie de la fondation du matérialisme dialectique par Marx et Lénine) ${ }^{35}$. Cette sélection de trois révolutions scientifiques est symptomatique : toutes ces sciences sont dogmaticodéductives ${ }^{36}$, au sens où pour Althusser, les sciences, « une fois qu'elles sont vraiment constituées et développées (...), n'ont nul besoin de la vérification de pratiques extérieures pour déclarer "vraies" c'est-à-dire connaissances, les connaissances qu'elles produisent ${ }^{37}$. Il est tout à fait révélateur que dans Lire Le Capital, pour penser l'intériorité des protocoles de vérification du matérialisme historique, Althusser mobilise, en guise d'exemple, la pratique théorique du mathématicien:

La "vérité " de son théorème lui est fournie à $100 \%$ par des critères purement intérieurs à la pratique de la démonstration mathématique, donc par le critère de la pratique mathématicienne, c'est-à-dire par les formes requises de la scientificité mathématique existante ${ }^{38}$.

Nous nous permettons de souligner: la vérité d'un théorème mathématique n'est pas renvoyée aux axiomes ou postulats dont il serait déduit, mais à la "pratique de la démonstration mathématique ». Par conséquent, si la conception althussérienne de la science est bien en un sens "formaliste » et "dogmatico-déductive", il nous semble quelque peu hardi d'en tirer la conclusion qu'elle formerait un système clos sur soi, où rien de neuf ne serait jamais produit en termes de vérité une fois posés les axiomes, constituant la véritable Origine d'un procès continu. Pour audacieuse que peut paraître cette thèse, nous pourrions suggérer que le modèle de la science althussérienne est celui de l'Ethica ordine geometrico demonstrata de Spinoza - héritier tout à la fois d'Euclide et de Descartes -, dont toute la forme, mais aussi le geste et le contenu sont profondément marqués par le modèle de la connaissance mathématique, qui est présent en particulier et de manière tout à fait centrale dans la critique du finalisme, ainsi que pour illustrer les trois genres de connaissance. Pour Spinoza, l'ordre d'exposition euclidien n'est pas une simple forme pour un contenu qui serait indifférent à celle-ci, et encore moins une prétention pour le système de se clore sur lui-même. Si la "manière des géomètres " s'impose à Spinoza, c'est en tant qu'elle procède synthétiquement et de manière productive, de la cause aux effets, par où Spinoza critique à la fois toute conception analytique du savoir et l'illusion finaliste et anthropocentrique, « en la remettant sur ses pieds $»^{39}$ Par conséquent, l'Éthique, œuvre systématique-dogmatique par excellence, ne peut être considérée comme un système analytique clos dont tout le développement et le contenu de vérité seraient déjà contenus dans les définitions et axiomes de départ, cette axiomatique faisant alors office d'Origine, de commencement absolu supposé tenir de l'évidence, et dont le développement dans les propositions et démonstrations ultérieures se ferait sans discontinuité ou production de vrai supplémentaire. La science de l'Éthique est au sens strict une production de connaissance, à la fois strictement interne au champ de la pensée théorique (l'attribut de la pensée), et immanente à l'activité du penser qui est productive et ne se contente pas d'expliquer ou de répéter un donné "tout fait " enveloppant en soi l'ensemble du procès (geste idéologique par excellence). Ainsi, pour Althusser, toute science, à la fois productive et synthétique, s'opposerait structurellement 
à la projection et à la répétition comme mécanismes spéculaires constitutifs de l'illusion idéologique subjectiviste et finaliste dont Spinoza fournit la première théorie critique et matérialiste dans l'Appendice au De Deo.

\section{Formalisme et activisme : Spinoza contre les technocrates}

19 "Reprendre et défendre (...) le mot "science" " sans "crie[r] à son simple énoncé au "positivisme" » exige de refuser "la représentation positiviste classique ou vulgaire, bourgeoise » de la science. À maintenir fort le lien entre science et émancipation, Althusser ne soutient aucunement que toutes les disciplines communément admises comme "scientifiques» soient par nature émancipatrices. Au contraire, dans les pratiques théoriques dominantes, en particulier dans le champ des sciences humaines, c'est bel et bien une idéologie technocratique de la science qui règne, instaurant un rapport de soumission spéculaire au Sujet de la science et réduisant le sujet à un assujetti, «bon sujet » ou « mauvais sujet » devant être réadapté. Mais, en toute rigueur, en vertu même de leur structure spéculaire et centrée autour d'un Sujet, ainsi que de leur opacité épistémologique et de leur instrumentalisme technocratique, ces disciplines ne sont pas des sciences au sens althussérien du terme. Toute accusation de "technocratisme élitiste » à l'égard de la pensée d'Althusser ne peut tenir qu'au prix d'une profonde occultation des nombreux passages où il en critique tous les représentants, tant dans le champ de la politique que dans celui des sciences. Dans «Philosophie et sciences humaines» (1963), s'inscrivant dans la lignée de Canguilhem, Althusser critique la non-scientificité de nombre de «sciences humaines » (psychologie, économie, sociologie, etc.) dont le flou régnant dans la définition de leur objet est rigoureusement indissociable des effets idéologiques et instrumentalisants qu'elles produisent sur les sujets. La critique épistémologique du caractère idéologique, non scientifique de ces « empirismes composites ", en fait simples " pratiques techniques », fait corps avec le constat de leur instrumentalisme au service d'un usage technocratique, « conditionnant » et " adaptant » les sujets " aux conditions existantes $»^{40}$. L'intervention philosophique d'Althusser vise précisément à creuser l'écart entre un tel instrumentalisme technocratique et la science marxiste qui est, par vocation, transformatrice, émancipatrice et révolutionnaire. La critique épistémologique entreprise par Althusser dans le champ des sciences humaines, visant à sauvegarder l'autonomie de la science en tant qu'elle exige la critique des tentations de resubjectivation-respécularisation, prendra un visage plus ouvertement politique dans des textes ultérieurs, dénonçant le technocratisme régnant de fait dans les organisations communistes, reproduisant la séparation «bourgeoise » entre « savants et ignorants » ${ }^{41}$.

La fonction de désubjectivation attribuée à la science, son caractère intrinsèquement émancipateur, n'a donc pas pour corollaire un technocratisme, tendance qu'Althusser combat vivement au sein du PCF. Il convient de souligner la conscience aigüe qu'a Althusser du risque que représente le technocratisme scientifique en politique: il va même jusqu'à reconnaître que la présence de la science marxiste-léniniste au cœur de l'idéologie prolétarienne risque toujours de se retourner en « simple argument d'autorité, c'est-à-dire comme un signe de reconnaissance ou un dogme, et à la limite, tout en étant proclamée la théorie du Parti, elle peut tout simplement disparaitre au profit d'une idéologie pragmatiste et sectaire qui ne sert plus que les intérêts de parti ou d'État. Il 
n'est pas besoin d'un long discours pour qu'on reconnaissance ici la situation présente, qui règne dans les partis marqués par la période stalinienne $»^{42}$.

Cet extrait révèle parfaitement la conviction althussérienne que la science ne peut devenir un dogme assujettissant, sous peine de «disparaitre " (le mot est souligné par l'auteur), c'est-à-dire de contredire sa propre essence. La raison profonde doit en être la suivante: la science est une activité (c'est bien l'idée qu'il y a derrière les mots de " procès » et de « pratique »), et n'est pas affaire de contenu théorique. En effet, on ne voit pas pourquoi un contenu scientifique disparaîtrait en étant traduit sous forme idéologique : transformé en mot d'ordre ou en incantation, son contenu ne change pas, du moins tant que l'on pense ce contenu comme une idée que l'on possède et que l'on se transmet.

À ce titre, nous pourrions à nouveau faire l'hypothèse d'un spinozisme althussérien. Pour Spinoza, la cause en vertu de laquelle l'idée vraie est adéquate n'est jamais extérieure à l'idée mais renvoie au procès de connaissance lui-même, qui marque l'idée vraie jusque dans sa forme intrinsèque. Pour l'auteur de l'Éthique, dont les mots ne sont pas sans faire songer à ceux employés par Marx en 1857-1858, l'idée n'est pas « quelque chose de muet comme une peinture sur un tableau" mais un "mode de penser", "à savoir le comprendre même (ipsum intelligere) $»^{43}$. L'idée vraie est ainsi génétiquement et formellement inséparable du processus causal de sa production. De même, la science pour Althusser n'est jamais réductible à un simple contenu, à une doctrine : si elle a bien à voir avec des «idées", c'est dans le sens d'un Spinoza caractérisant l'idée comme rigoureusement indissociable de l'activité par où elle est produite. C'est dire que la scientificité de la science tient bien davantage à une question de forme, de structure de son activité. Si la science peut disparaitre, et si elle est justement en train de disparaître pour Althusser en 1969, ce n'est pas comme contenu doctrinal, mais comme pratique désubjectivante, activité de transformation du donné idéologique. Liée à sa position «formaliste internaliste », d'après laquelle la vérité d'une connaissance est formelle et intrinsèque, se confondant «totalement avec les formes rigoureuses de l'exercice de la pratique scientifique considérée $»^{44}$, nous pourrions ainsi entrevoir chez Althusser une posture «activiste» ou "expérientielle» de la pratique théorique. L'horizon inséparablement théorique et politique de l'intervention althussérienne pourrait alors s'exprimer dans les mots d'é. Balibar décrivant la «stratégie de libération collective » de Spinoza : « être le plus nombreux possible à penser le plus possible ${ }^{45}$.

\section{Conclusion}

Renouer avec le sens politique du théoricisme exige de soutenir que la science et sa différence d'avec l'idéologie ne sont pas affaire de contenu, mais de structure pratique. "Servir le peuple », ou tenir un discours matérialiste, c'est mettre en place un processus qui a pour effet de fissurer le discours de l'idéologique, sa clôture sur soi, son "narcissisme ${ }^{46}$ structurel, ainsi que les rapports spéculaires entre ses bons et mauvais, grands et petits sujets. C'est dire que l'idée de coupure épistémologique reste incontournable, à condition de bien la comprendre. Si l'idéologie peut bien être émancipatrice pour Althusser (notamment dans sa fonction d'interpellation du «sujetmilitant $»^{47}$ ), ce n'est pas dans le sens où la science peut l'être (effet de désubjectivation par déspécularisation et décentrement de la structure d'interpellation). À abandonner toute idée de coupure, on se condamne à faire jouer idéologie contre idéologie, classe 
contre classe, identité contre identité. La leçon intempestive à tirer d'une réhabilitation du théoricisme comme stratégie politique pourrait être la suivante : il est incontournable de penser un moment de rupture dans le processus imaginaire d'identification, qui soit le produit du travail asubjectif du concept qui « fait le vide » du sujet.

Cette thèse se révèle centrale pour comprendre l'intervention philosophique qu'Althusser a voulu tout à la fois pratiquer et théoriser. La «nouvelle pratique de la philosophie » n'est ni en rapport d'exploitation vis-à-vis de la science, ni en rapport de soumission à l'égard de l'idéologie dominante. Mais elle ne peut non plus se limiter à une fonction gramscienne d'unification et ce, quand bien même il s'agirait d'unifier l'idéologie prolétarienne. Au contraire, on verra se développer de plus en plus chez Althusser le thème de la "guérilla philosophique " comme antidote à la pensée-système, ce «lien subtil, mais très fort, qui relie l'État à la philosophie $»^{48}$. Le passage $d^{\prime}$ " un rapport de servitude et d'exploitation" à un «rapport de libération et de liberté ${ }^{49}$ entre philosophie, idéologies et pratiques ne semble pouvoir se passer de l'effet de désubjectivation, de décentrement et de déspécularisation caractérisant la pratique scientifique. Autrement dit, il ne peut s'agir d'opposer unilatéralement les idéologies bourgeoises et prolétariennes, qui sont dans un rapport d'inégalité qualitative. Le refus althussérien de la théorie lyssenkiste des deux sciences prend alors une signification toute politique: si la science est du côté du prolétariat, l'idéologie de ce dernier, «travaillée " par les idées vraies de la première, se révèle " tout autre », précisément en vertu de cette présence du scientifique en elle ${ }^{50}$. De ce point de vue, tout l'enjeu des interventions théoriques et politiques d'Althusser est précisément de dénoncer le fait que les organisations du mouvement ouvrier se sont "prises au jeu» des formes de lutte bourgeoise, non seulement au niveau de la lutte politique étatico-parlementaire, mais également au niveau de ses formes de pratique théorique et idéologique, en se limitant de facto à un travail d'unification et de diffusion de la Vérité du Parti auprès des masses ${ }^{51}$, perdant de vue la tâche, à la fois théorique et pratique, de décentrement et de déspécularisation au cœur du projet révolutionnaire marxiste.

La question qui reste ouverte, ou plutôt qui s'ouvre avec insistance à partir de là, est celle des formes concrètes d'organisation, de production et de circulation du savoir, qui conditionnent directement les potentialités émancipatrices, c'est-à-dire l'existence même, de la science. De même, notre présente contribution gagnerait à se prolonger dans une analyse plus rigoureuse du rôle spécifique de la philosophie comme pratique théorique distincte, jouant le rôle de «tierce instance » entre science et politique. Enfin, s'il est patent que la pratique psychanalytique joue un rôle paradigmatique dans la manière dont Althusser entreprend de refonder le concept de science en tant que pratique de transformation (a)subjective, de décalage et de déplacement ${ }^{52}$, il serait nécessaire d'étudier plus précisément ce qui rapproche et distingue la pratique théorique (scientifique et philosophique) de la pratique analytique, ou encore de l'artistique ${ }^{53}$. 


\section{NOTES}

1. «Philosophie et révolution. Althusser sans le théoricisme : entretien avec G. M. Goshgarian », in Période, 2015, consulté la dernière fois le 22 mars 2015. URL: http://revueperiode.net/ philosophie-et-revolution-althusser-sans-le-theoricisme-entretien-avec-g-m-goshgarian/.

2. L. Althusser, "Éléments d'autocritique", in Solitude de Machiavel et autres textes, Paris, PUF, 1998, p. 163. L'image léninienne de la "courbure du bâton ", "simple formule " qui "paraît contenir toute une théorie de l'efficace du vrai », fait de nombreuses apparitions dans l'œuvre d'Althusser. Cf. notamment la "Soutenance d'Amiens ", in Solitude de Machiavel, op. cit., p. 205 ; « Marx dans ses limites », in Écrits philosophiques et politiques, t. I, Paris, Stock/IMEC, 1994, p. 387.

3. Bien entendu, il ne pourrait s'agir de se contenter d'un renversement unilatéral de cette lecture téléologique, au profit d'une posture (non moins idéologique) consistant à soutenir que «tout Althusser » serait déjà donné dès l'origine, et que ses multiples rectifications autocritiques n'auraient rien ajouté ou infléchi en termes de tendances.

4. L. Althusser, « Éléments d'autocritique », in Solitude de Machiavel, op. cit., p. 176.

5. Ibid., p. 171.

6. L. Althusser, Lire « Le Capital », Paris, PUF, 2008, p. 22.

7. Ibid., p. 340-341. Cf. également le texte ronéotypé de 1965 intitulé «Théorie, Pratique théorique et Formation théorique. Idéologie et lutte idéologique ", cité dans les notes d'édition, in L. Althusser, Écrits philosophiques et politiques, t. I, op. cit., p. 528. Il existe une traduction anglaise de ce texte demeuré inédit en français: "Theory, Theoretical Practice and Theoretical Formation: Ideology and Ideological Struggle ", in Philosophy and The Spontaneous Philosophy of the Scientists \& Other Essays, London/New-York, Verso, 1990. Le texte anglais est intégralement consultable en ligne. URL : http://www.marx2mao.com/Other/PSPS90.html (consulté la dernière fois le 02 avril 2015).

8. Cf. "Sur l'évolution du jeune Marx» (1970), in L. Althusser, Éléments d'autocritique, Paris, Hachette, 1974, p. 103-126; " Note sur les AIE », in L. Althusser, Sur la reproduction, Paris, PUF, 2011, p. 260 ; «Éléments d'autocritique» (1974), in Solitude de Machiavel, op. cit., p. 174 ; "Sur Marx et Freud» (1976), in Écrits sur la psychanalyse, Paris, Stock/IMEC, 1993, p. 228-232 ; " Marx dans ses limites " (1978), in Écrits philosophiques et politiques, Tome I, op. cit., p. 381-397 ; «Le marxisme aujourd'hui » (1978), in Solitude de Machiavel, op. cit., p. 299.

9. L. Althusser, «La philosophie comme arme de la révolution », in Solitude de Machiavel, op. cit., p. 156.

10. L. Althusser, «Éléments d'autocritique », in Solitude de Machiavel, op. cit., p. 171.

11. É. Balibar, «Préface à la nouvelle édition. Du marxisme althussérien aux philosophies de Marx ? Vingt ans après », in La philosophie de Marx, Paris, La Découverte, 2014, p. 16.

12. L. Althusser, «La philosophie comme arme de la révolution », in Solitude de Machiavel, op. cit., p. 155.

13. J. Rancière, La leçon d'Althusser, Paris, La Fabrique, 2011, p. 233.

14. L. Althusser, «Soutenance d'Amiens », in Solitude de Machiavel, op. cit., p. 205-206.

15. L. Althusser, L'avenir dure longtemps, Paris, Stock/IMEC, 2007, p. 483-484.

16. Cf. Fabio Bruschi, «Faire le vide. Intervention intellectuelle et idéologie entre Spinoza et Althusser ", in Cahiers marxistes, $n^{\circ}$ 244, février-mars 2015, p. 67-83.

17. L. Althusser, «La philosophie comme arme de la révolution », in Solitude de Machiavel, op. cit., p. 155.

18. Ce point sera davantage explicité dans la section suivante (point 3). 
19. L. Althusser, Sur la reproduction, op. cit., p. 262 ; L. Althusser, Sur la philosophie, Paris, Gallimard, 1994, p. 171.

20. L. Althusser, Sur la reproduction, op. cit., p. 221.

21. Cf. la section "Processus de la pratique théorique ", in L. Althusser, Pour Marx, Paris, La Découverte, 2005, p. 187-197.

22. L. Althusser, Lire « Le Capital », op. cit., p. 41.

23. L. Althusser, Initiation à la philosophie pour les non-philosophes, Paris, PUF, 2014, p. 168.

24. L. Althusser, Lire «Le Capital », op. cit., p. 41.

25. É. Balibar, «L'objet d'Althusser », in Sylvain Lazarus (dir.), Politique et philosophie dans l'œuvre de Louis Althusser, Paris, PUF, 1993, p. 98.

26. Nous pensons en particulier à La leçon d'Althusser de J. Rancière. Nous ne nions pas la légitimité et la productivité d'une telle critique, à condition de noter qu'elle prend pour objet les " effets objectifs» produits par la position althussérienne dans une conjoncture donnée (J. Rancière, La leçon d'Althusser, op. cit., p. 254, note 1). Indépendamment de ces derniers, l'intervention althussérienne se destinait bien à "un rôle libérateur " des pratiques, tant théoriques que politiques (Cf. L. Althusser, « La transformation de la philosophie. Conférence de Grenade (1976) ", in Sur la philosophie, op. cit., p. 177 ; «Lénine et la philosophie », in Solitude de Machiavel, op. cit., p. 143). Si Althusser avait bien conscience du risque jamais écarté, sinon de la nécessité pour toute prise de position de dévier sous l'effet de la conjoncture opaque et complexe dans et sur laquelle elle intervient, indépendamment de toute intention subjective, il permet également de penser l'irréductibilité de toute position à son effectuation historico-concrète en conjoncture, c'est-à-dire la « répétabilité » et la rectification de sa tendance. Cf. la section «Des tendances en philosophie » dans les "Éléments d'autocritique », in Solitude de Machiavel, op. cit., pp. 189-195. Cf. également la section "Une politique "finie": déviation et dialectique », in A. Cavazzini, Crise du marxisme et critique de l'État. Le dernier combat d'Althusser, Séminaire du GRM 2007-2008, Séance du 16 février 2008. URL : http://f.hypotheses.org/wp-content/blogs.dir/1106/ files/2013/01/GRM_1_annee_Cavazzini_Althusser.pdf. Notons enfin que, malgré ses critiques, Rancière reconnaît que le moment théoriciste a constitué, dans la conjoncture du milieu des années 1960, le moment le plus émancipateur de la trajectoire althussérienne, en particulier par son exigence d'une « autonomie du théorique " vis-à-vis du PCF (J. Rancière, La leçon d'Althusser, op. cit., p. 93-99).

27. La problématique (ou Généralité II) est définie comme «le corps des concepts dont l'unité plus ou moins contradictoire constitue la "théorie" de la science au moment (historique) considéré » (L. Althusser, Pour Marx, op. cit., p. 188).

28. A. Badiou, «Althusser : le subjectif sans sujet », in Abrégé de métapolitique, Paris, Seuil, 1998, p. 67.

29. K. Marx, « Grundrisse der Kritik der politischen Ökonomie » (1857-1858), in Philosophische und ökonomische Schriften, Stuttgart, Reclam, 2008, p. 99.

30. L. Althusser, "Trois notes sur la théorie des discours ", in Écrits sur la psychanalyse, op. cit., p. 142 ; Sur la reproduction, op. cit., pp. 216-220.

31. Dans ses "Éléments d'autocritique" Althusser rectifie la thèse de la coupure en la « politisant », tout en maintenant sa validité (Cf. Solitude de Machiavel, op. cit., p. 163-165).

32. L. Althusser, « Le marxisme aujourd'hui », in Solitude de Machiavel, op. cit., p. 303.

33. L. Althusser, « Lénine et la philosophie », in Solitude de Machiavel, op. cit., p. 134.

34. L. Althusser, Sur la reproduction, op. cit., p. 231.

35. Ibid., pp. 47-52.

36. De là à conclure qu'une telle science devient un système clos c'est-à-dire, précisément, idéologique, il n'y a qu'un pas que nous nous garderons bien de franchir, comme nous allons essayer de le montrer ci-après.

37. L. Althusser, Lire Le Capital, op. cit., p. 65. 
38. Ibid., p. 65-66.

39. P. Macherey, Hegel ou Spinoza, Paris, La Découverte, 1990, p. 70-71.

40. L. Althusser, "Philosophie et sciences humaines ", in Solitude de Machiavel, op. cit., p. 52-55 ; voir aussi Pour Marx, op. cit., p. 171-172.

41. L. Althusser, "Marx dans ses limites ", in Écrits philosophiques et politiques, Tome I, op. cit., p. 389 ; L. Althusser, Sur la reproduction, op. cit., p. 259-260 ; L. Althusser, $22^{e}$ congrès, Paris, Maspero, 1977, pp. 66-67 ; L. Althusser, Ce qui ne peut plus durer dans le parti communiste, Paris, Maspero, 1978, p. 20-30.

42. L. Althusser, Sur la reproduction, op. cit., p. 259-260.

43. Baruch Spinoza, Éthique, traduit du latin par B. Pautrat, Paris, Seuil, 1999, Partie II, Proposition XLIII, Scolie, p. 181.

44. L. Althusser, Lire «Le Capital », op. cit., p. 66.

45. É. Balibar, Spinoza et la politique, Paris, PUF, 1984, p. 118.

46. « Dans le mode de production théorique de l'idéologie (tout différent, sous ce rapport, du mode de production théorique de la science), la formulation d'un problème, n'est que l'expression théorique des conditions permettant à une solution déjà produite en dehors du processus de connaissance, parce qu'imposée par des instances et exigences extra-théoriques (par des "intérêts" religieux, moraux, politiques ou autres), de se reconnaître dans un problème artificiel, fabriqué pour lui servir tout à la fois de miroir théorique et de justification pratique» (L. Althusser, Lire «Le Capital», op. cit., p. 56). Ainsi, l'idéologie est caractérisée par une structure spéculaire de reconnaissance, dont les réponses imposées d'avance par des intérêts extrathéoriques «se reconnaissent » dans la production de questions elles-mêmes mystifiées, de sorte qu'elles ne puissent à leur tour que se reconnaître dans les solutions imposées. «Dans l'idéologie toutes les questions sont ainsi réglées d'avance, par essence (...). Les questions y sont donc des questions feintes qui ne sont que le reflet spéculaire des réponses qui préexistent aux questions » (L. Althusser, "Trois notes sur la théorie des discours ", in Écrits sur la psychanalyse, op. cit., p. 137). Aux antipodes de ce narcissisme spéculaire et clos sur soi, la science est décrite comme un «cercle ouvert", fonctionnant "comme un dispositif conceptuel "de base" ouvert sur "l'infinité" (Lénine) de son objet, c'est-à-dire destiné à poser et affronter sans cesse des problèmes pour produire sans cesse de nouvelles connaissances » (L. Althusser, « Sur l'évolution du jeune Marx ", in Éléments d'autocritique, op. cit., p. 111).

47. L. Althusser, Sur la reproduction, op. cit, p. 259.

48. L. Althusser, Initiation à la philosophie pour les non-philosophes, op. cit., p. 370.

49. Ibid., p. 372.

50. Cf. notamment L. Althusser, Sur la reproduction, op. cit., p. 258-262.

51. L. Althusser, $22^{e}$ congrès, op. cit., p. 66-67 ; L. Althusser, Ce qui ne peut plus durer dans le parti communiste, op. cit., p. 20-30.

52. Pour une esquisse d'un rapprochement entre la pratique théorique matérialiste et la cure analytique, nous nous permettons de renvoyer à J. Matthys, «Critique et clinique. La pratique analytique comme modèle opératoire pour l'intervention philosophique chez Althusser ", in Meta: Research in Hermeneutics, Phenomenology, and Practical Philosophy, 2014, Vol. VI, $\mathrm{n}^{\circ} 1$, p. 266-287.

53. Cf. la quatrième section «Écrits sur l'art ", in L. Althusser, Écrits philosophiques et politiques, t. II, Paris, Stock/IMEC, 1997, p. 551-620, reprenant notamment « Sur Brecht et Marx », « Lettre sur la connaissance de l'art » et « Cremonini, peintre de l'abstrait ». 


\section{RÉSUMÉS}

L'objectif de cet article est d'esquisser une réhabilitation du théoricisme althussérien compris comme stratégie politique. À cette fin, nous étudions la manière dont la pratique théorique est supposée, en vertu de sa structure spécifique, produire des effets émancipateurs au sein de la lutte des classes. Ce faisant, nous découvrirons que le cœur du projet philosophique d'Althusser, avant comme après l'autocritique, consiste à défendre autant qu'à transformer radicalement le concept de science, afin de pouvoir rendre compte de son autonomie et de son efficace, relatives mais bien réelles, dans la structure sociale conflictuelle. Contre l'idée reçue selon laquelle l'althussérisme ne peut aboutir qu'à une posture technocratique de maîtrise chargeant les scientifiques d'éclairer les masses en leur transmettant des idées vraies, nous défendons que la science doit avant tout être comprise comme pratique productive et travail asubjectif des concepts, auquel chacun est appelé à prendre part afin de faire l'expérience d'une dessaisie de soi comme sujet - par où s'ouvrent de nouvelles voies de subjectivation, en lien avec de nouvelles formes de vie et d'organisation.

\section{INDEX}

Thèmes : philosophie politique, épistémologie, philosophie française contemporaine, matérialisme, structuralisme, marxisme

Index chronologique : années soixante, années soixante-dix Index géographique : France

Mots-clés : Althusser, science, savoir, émancipation, idéologie, sujet, subjectivité, Rancière Jacques, Spinoza

Keywords : Althusser Louis, knowledge, ideology, subject, subjectivity

\section{AUTEUR}

\section{JEAN MATTHYS}

Jean Matthys a étudié la philosophie et les sciences politiques aux Facultés universitaires SaintLouis (Belgique) et est titulaire d'un Master en philosophie obtenu à l'Université catholique de Louvain (Belgique). Ses recherches sont consacrées aux rapports entre spinozisme et marxisme au XX $X^{\mathrm{e}}$ siècle.Courriel : jean_matthys@hotmail.com 